\title{
Electrostatic attraction caused by triboelectrification in climbing geckos
}

\author{
Yi SONG, Zhouyi WANG, Yang LI, Zhendong DAI* \\ College of Mechanical and Electrical Engineering, Nanjing University of Aeronautics and Astronautics, Nanjing 210016, China \\ Received: 27 November 2019 / Revised: 10 February 2020 / Accepted: 20 March 2020 \\ (C) The author(s) 2020.
}

\begin{abstract}
Adhesion achieved through feet setae is fundamental for gecko agilely maneuvering. Although diverse hypotheses have been proposed, none of them thoroughly explains the setae function, implying a kind of hybrid-mechanism-based adhesion in geckos. In addition to van der Waals interactions and capillary force, the electrostatic attraction that emerges from triboelectrification was suggested as a component of setae adhesion. Nevertheless, the contribution by electrostatic attraction to the total setae attachment is still controversial. In this study, we analyzed the occurrence of electrostatic attraction at gecko setae through experiments and model analyses. By touching the substrates with only $\sim 1 / 70^{\text {th }}$ of the foot area, freely wall-climbing geckos developed tribocharge at their feet setae with a density of $\sim 277$ $\mathrm{pC} / \mathrm{mm}^{2}$, generating electrostatic attractions with a strength of $\sim 4.4 \mathrm{mN} / \mathrm{mm}^{2}$. From this perspective, the adhesion driven by triboelectrification could account for about $1 \%$ of total adhesion. Model analyses at spatula level indicated a similar result showing that the electrostatic force might account for $\sim 3 \%$ of the adhesion that facilitates wall-climbing in geckos. The low contribution of the electrostatic force partly explains why geckos always face difficulty in maneuvering onto those substrates (e.g., teflon) where they could easily develop tribocharge but difficultly generate van der Waals force. However, long-range electrostatic forces may play other roles in a distance range where the van der Waals interaction cannot function. These findings not only add to our understanding of the mechanism of gecko adhesion, but also will help us advance gecko-inspired fibular adhesives.
\end{abstract}

Keywords: biotribocharge; electrostatic force; geckos; adhesion

\section{Introduction}

Geckos are recognized as one of the most excellent climbers that can maneuver on various terrains in all orientations [1-5]. Their stride frequencies can be as large as a dozen Hertz, and their speeds could be as fast as $\sim 1 \mathrm{~m} / \mathrm{s}$. Previous studies have attributed the versatility of geckos in locomotion to their abilities to alter body dynamics and rapidly obtain proper adhesion through their compliant, hierarchical, and hairy footpads [6-8]. Therefore, understanding the physics of geckos' feet and the mechanism of adhesion generation is of great significance to understand the biological attachment mechanism and inspire artificial adhesives.

Attempts aiming at determining the adhesion mechanisms of geckos can be traced back to the 1800s. In the last two centuries, many hypotheses, including adhesive secretion, suction, friction, and micro-interlocking, have been proposed to explain the adhesion mechanism of geckos [9-12], but were not supported by experimental observations [13-16]. In 1900, Haase [17] proposed that geckos probably adhere through intermolecular forces. Hiller [18] further

* Corresponding author: Zhendong DAI, E-mail: zddai@nuaa.edu.cn 
found that the setae can stick to hydrophilic surfaces with high surface energies but cannot adhere to hydrophobic surfaces with low surface energies, confirming that the gecko adhesion involves intermolecular interactions which are related to the surface energies [19]. After separately considering the effects of the polarizability and hydrophobicity of substrate on setae adhesion, Autumn et al. [16, 20] rejected other proposals but supported that the van der Waals force is the primary source of setae adhesion, allowing a single seta of a gecko to generate shear and normal forces for 200 and $40 \mu \mathrm{N}$, respectively [20]. However, Huber et al. [21] found that the adhesive force of the spatulae increases with the humidity and declared that the capillary force (or capillary-related force) must also contribute to gecko adhesion, although Refs. [22-24] support that the high humidity softens the setae rather than inducing capillary force. Because a seta can only generate adhesion if it is pushed toward a substrate then slid for a short distance [20], and its main component ( $\beta$-keratin [25]) is easily positively charged while touching other materials [26, 27], the electrostatic force that emerges from the tribocharge was also reasonably considered as a potential source of setae adhesion [10]. According to the findings in triboelectrification [28-31], the strength of electrostatic adhesion is determined by the properties of setae and substrate but urgently requires accurate quantifying in geckos.

Prevenslik [32] theoretically analyzed the possibility of electrostatic adhesion in geckos but failed to provide experimental evidence. Izadi et al. [33] studied the adhesion of a kind of gecko-inspired adhesive made with teflon amorphous fluoropolymer (teflon AF) and confirmed the electrostatic adhesion in fibrous adhesives. They also studied the triboelectrification on gecko feet by sliding a gecko foot on a teflon AF film, obtaining a considerable amount of tribocharge, which was capable of generating an attraction twice the shear force they measured [34]. Therefore, they drew a well-reasoned conclusion claiming that the attraction driven by electrostatic interactions instead of the van der Waals force is the major source of gecko adhesion. However, as they mentioned, the hairs and teflon are almost the easiest to be tribocharged according to the tribocharge series [26, 27], but nearly the most difficult to generate van der Waals adhesion [35]. Thus, the tribocharge measured by sliding geckos feet on teflon seemed to be overlarge whereas the force seemed to be insufficient, compared with running geckos on other materials. In our previous work [36], we measured the tribocharge at the geckos' feet when they were freely ascending an acrylic oligomer film and found a much lower charge [37], but failed to estimate the exact contribution of the tribocharge because of the unknown actual contact charge density. To accurately determine the charge density, and thereby electrostatic force, synchronous measurements of the tribocharge and contact area are required but currently almost impossible.

In this study, we took a step forward by comprehensively considering the contact, triboelectrification, and adhesion. Through experiments and experimentbased model analyses, we estimated the contribution of electrostatic force originating from triboelectrification to setae adhesion in freely climbing geckos. This study will not only provide an insight into the setae adhesion in geckos, but also help us advance the gecko-inspired fibrous adhesives.

\section{Materials and methods}

\subsection{Animals}

Five geckos (Gekko gecko) with masses of $78.3 \pm 14.4 \mathrm{~g}$ were used in the experiments. They were purchased from Guangxi, China, raised in a temperature range of $26-28{ }^{\circ} \mathrm{C}$ and humidity of $55 \%-65 \%$. The room was illuminated for $12 \mathrm{~h}(7 \mathrm{am}-7 \mathrm{pm})$ every day. The geckos were provided crickets every two days and water daily.

The experiment was approved by the Jiangsu Association for Laboratory Animal Science and performed following the Guide of Laboratory Animal Management Ordinance of China. No animals were hurt during the experiments.

\subsection{Experimental setup}

As mentioned earlier, precisely estimating the electrostatic adhesion that emerges from triboelectrification requires an accurate and synchronous measurement 
of the tribocharge, contact area, and reaction forces at the feet of geckos, which is almost impossible by using current techniques. Therefore, in this study, we used data obtained from two separate experiments, as described in Sections 2.2.1 and 2.2.2.

\subsubsection{Synchronous measurement of tribocharge and reaction force}

Zou et al. [26] established a standard for the measurement of contact charges. By using a similar principle to measure the tribocharge and measuring contact force via force sensors[, we obtained the synchronous tribocharge and contact force at the feet of geckos when they climbed a substrate covered with acrylic oligomer film [36]. In this study, we used the charge data adopted from our previous work (Ref. [36]).

\subsubsection{Synchronous measurement of contact area and reaction force}

As shown in Fig. 1, an aisle was constructed using three-dimensional (3D) force sensors and clear acrylic sheets $(160 \mathrm{~mm} \times 60 \mathrm{~mm} \times 3 \mathrm{~mm})$. The contact angle of the acrylic sheet $\left(68.1^{\circ}\right)$ is similar to that of the substrate $\left(64.8^{\circ}\right)$ used in measuring the tribocharge so that the chemistries of the substrates do not significantly change the adhesion performance according to Hiller's findings [18]. Light strips were appended to the acrylic sheet to form frustrated total internal reflection (FTIR [37]) that can highlight the contact regions once the geckos touched the acrylic. Two baffles were used to constrain the geckos, a dust removal film was placed at the start of the aisle to clean the feet of geckos, and a darkened box was placed at the end of the aisle to induce the geckos to run. While the geckos climbed upward, we collected the force signals using a data acquisition (DAQ) module (National Instrument Inc., Texas, USA) at $1,000 \mathrm{~Hz}$ and monitored their climb from the back using a highspeed camera (500 fps, Ispeed-3, Olympus, Japan).

The experiments were conducted at the temperature of $\sim 25{ }^{\circ} \mathrm{C}$ and the humidity of $\sim 55 \%$. The animals ran at most three times every two days. They were allowed to rest for at least $1 \mathrm{~h}$ before running. The trials in which the animals suddenly stopped, speeded up, turned back, or touched the baffles

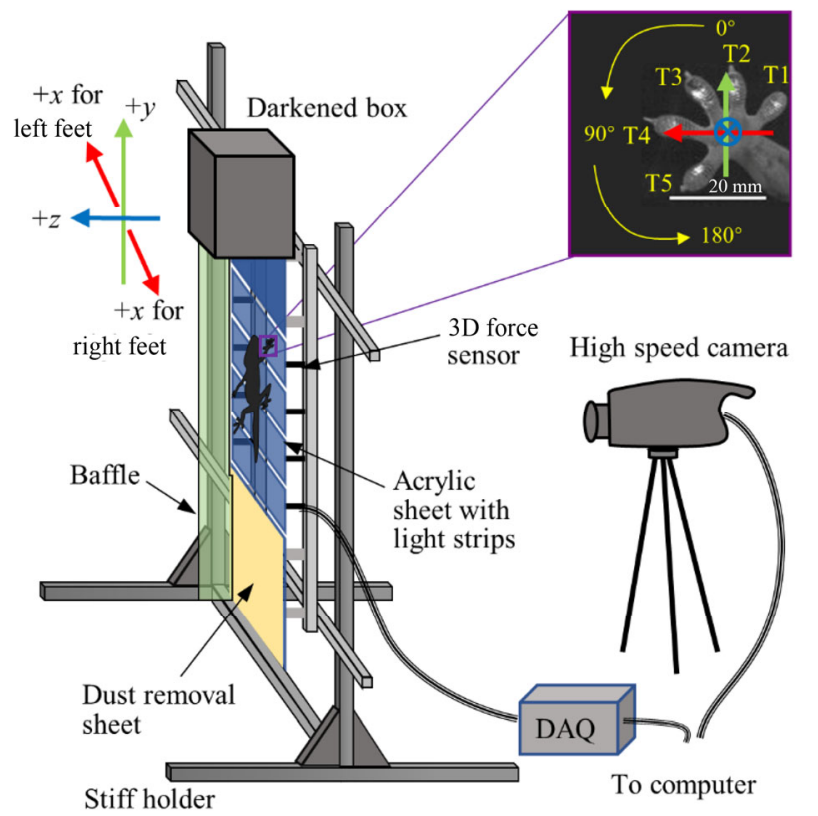

Fig. 1 Approach to synchronously measure the contact area and reaction force at the feet of climbing geckos. The coordinate system at the top-left indicates the positive directions for measured force. In particular, the lateral force (i.e., the force in $x$-direction) was set to be positive when it pointed to the outward as shown by the top-right inset, and the toes at the left feet were counted anticlockwise while those at the right feet were counted clockwise.

were discarded. Finally, we obtained seven trials from each gecko.

\subsection{Data processing}

The highspeed videos were processed using MATLAB 2018 (MathWorks Inc., Massachusetts, USA). Through image processing [37], the exact contact area $\left(A_{i}\right)$ and orientation $\left(\phi_{i}\right)$ of gecko toes were calculated. In particular, by considering the bilateral symmetries of their limbs and the reaction force, the outward lateral force was set as positive (as shown by the coordinate system in Fig. 1), and the toe orientation calculated relative to the upward direction was counted anticlockwise and clockwise for the left and right feet, respectively.

Two methods were applied to calculate the adhesive strength of the feet of the geckos. First, the overall contact area was used, as in our previous work [36]. Second, the resultant contact area, which was calculated by regarding the direction of toes as vectors, was applied. To compute the resultant contact area, we used 


$$
\begin{aligned}
& A_{x}=\sum_{i=1}^{5} A_{i} \sin \varphi_{i} \\
& A_{y}=\sum_{i=1}^{5} A_{i} \cos \varphi_{i} \\
& A_{\mathrm{s}}=\sqrt{A_{x}^{2}+A_{y}^{2}}
\end{aligned}
$$

where $A_{x}$ and $A_{y}$ are the resultant contact areas in $x$ - and $y$-directions, respectively; and $A_{\mathrm{s}}$ is the resultant contact area concerning the shear force.

SPSS 19.0 (IBM, NY, USA) was used to perform the statistical analysis (general linear regression analysis and ANOVA) with a significance level of 0.05 used throughout the analysis.

\section{Results}

\subsection{Nominal tribocharge density}

As shown in Fig. 2(a), when geckos ascended the vertical wall and treaded on the acrylic oligomer film, we obtained the tribocharge and reaction force at their feet [36]. The result indicated that the tribocharge was proportional to the frictional adhesion, as both increased with the nominal contact area (Fig. 2(b)). Consequently, we found a nominal shear strength of $\sim 1.9 \mathrm{mN} / \mathrm{mm}^{2}$ and a nominal charge density of $\sim 4.05 \mathrm{pC} / \mathrm{mm}^{2}$ by dividing the measured force and charge with the nominal contact area. However, we failed to obtain the exact contact area for accurately estimating the exact contribution of the tribocharge, and could therefore determine a very tiny amount of electrostatic force by using the model shown in Fig. 2(c).

\subsection{Real contact area and reaction force}

As shown in Fig. 3(a), we measured the real contact area at the feet of the geckos through FTIR when they vertically climbed on the acrylic aisle. While contacting the substrate, the geckos did not concentrate adhesion to a specific toe but distributed it to multiple toes differing in orientations (Figs. 3(a) and $3(\mathrm{~b}))$. The maximum overall actual contact area was about $4-6 \mathrm{~mm}^{2}$ at a whole foot, much smaller than the nominal area of a gecko foot (Fig. $3(b))$. Assuming that the toes function as vectors (Fig. 3(b)), we obtained the resultant contact area
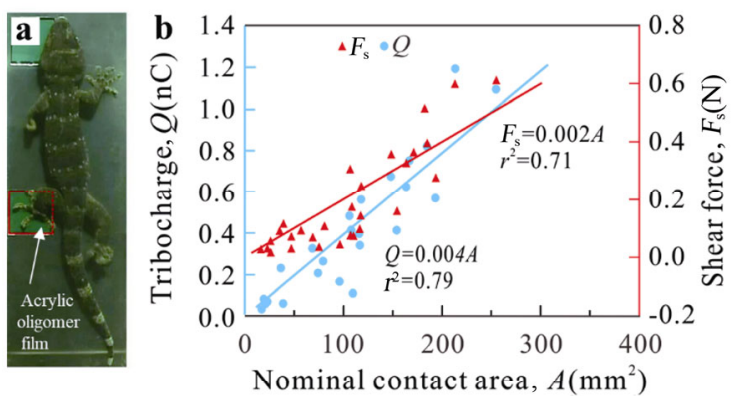

c

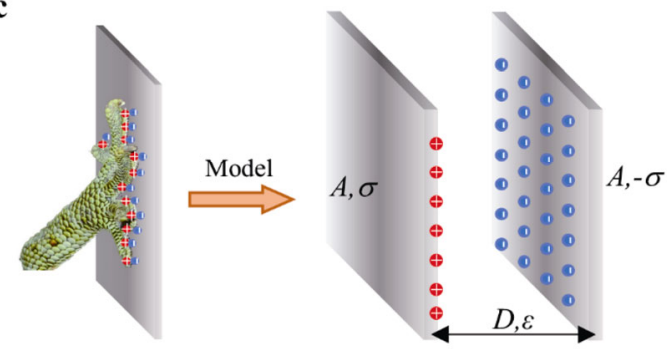

Fig. 2 Tribocharge and adhesion in climbing geckos. The data was adopted from our previous work [36]. (a) The interaction between a gecko foot and an acrylic oligomer film; (b) the tribocharge and adhesion are related to the nominal contact area; and (c) a model that describes the electrostatic force between a gecko foot and the substrate. $A$, contact area; $\sigma(-\sigma)$, charge density; $D$, distance between foot and substrate; and $\varepsilon$, electric inductivity.
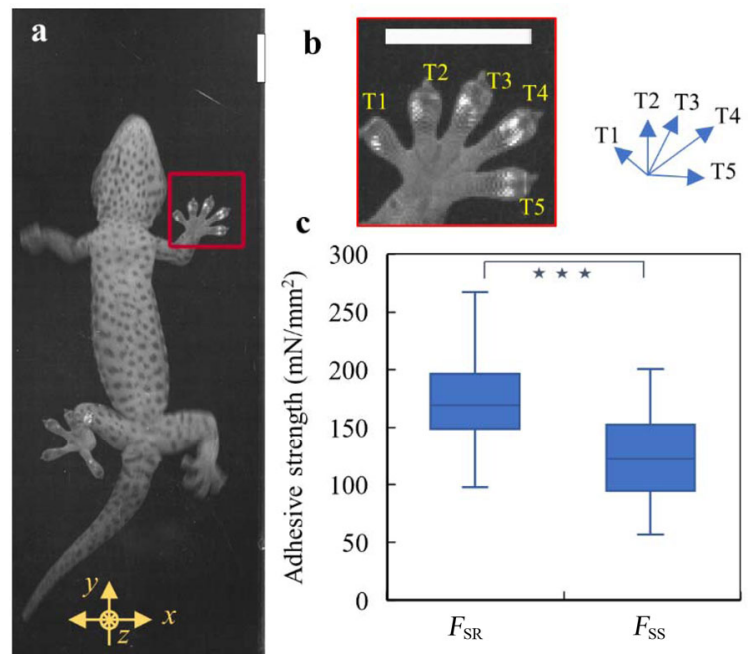

Fig. 3 Estimation of real contact area and reaction force at the feet of climbing geckos. (a) The measurement of contact area at the feet of a gecko when climbing an acrylic board; (b) distributed toes functioning as vectors; and (c) the frictional adhesion strength calculated using resultant contact area $\left(F_{\mathrm{SR}}\right)$ and overall contact area $\left(F_{\mathrm{SS}}\right)$. $(\mathrm{a}, \mathrm{b})$ The white parts show the contact regions. The scale bars are $20 \mathrm{~mm}$, and the resultant contact area was calculated by regarding toe contacts as vectors with angles of $\varphi_{i}$ and values of $A_{i}$, as shown in (b). Significance level: $\star P \geqslant 0.05$; $\star \star \quad P \geqslant 0.01 ; \star \star$ $\star P<0.01$. 
at the feet by Eq. (1). The general linear regression analysis of the contact area and adhesion at midstance indicated that the adhesion $(F)$ was proportional to $A_{\mathrm{s}}$ by $F=164.1 A_{\mathrm{s}}\left(r^{2}=0.71\right.$, linear regression, $P<$ $0.01)$. We calculated a shear strength $\left(F_{\mathrm{SR}}\right)$ of $172.6 \pm$ $36.3 \mathrm{mN} / \mathrm{mm}^{2}$, a result agreeing with another test [5], by dividing the force with the resultant contact area, as shown in Fig. 3(c). However, the result $\left(F_{\mathrm{SS}}\right)$ reduces to $130.3 \pm 32.3 \mathrm{mN} / \mathrm{mm}^{2}$, when using the overall contact area $\left(\Sigma \mathrm{A}_{i}\right)$ instead (Fig. 3(c); ANOVA, $P<0.01)$.

\section{Discussion}

\subsection{Exact tribocharge density}

Hiller [18] determined that the gecko adhesion is highly dependent on the surface energies of the substrates. As the acrylic board in our experiment \#2 possesses a similar contact angle (surface energy) with the acrylic oligomer film in our experiment \#1, we assumed that the chemistry of the substrates would not significantly affect the adhesion. Furthermore, according to Huber et al. [38], the surface irregularity of the acrylic oligomer film $(\sim 500 \mathrm{~nm})$ does not significantly weaken the setae adhesion either. The temperatures and humidity in both experiments were similar. Therefore, we believed that the geckos possess similar characteristics in frictional adhesion and triboelectrification on both substrates.

By comparing the nominal $\left(1.9 \mathrm{mN} / \mathrm{mm}^{2}\right)$ and the exact $\left(130.3 \mathrm{mN} / \mathrm{mm}^{2}\right)$ adhesion strengths, we found that the real adhesion strength is $\sim 69$ times that of the nominal one, indicating that the real contact area at a foot is about $1 / 69^{\text {th }}$ of its whole area $\left(\sim 250 \mathrm{~mm}^{2}\right.$ in our experiments). This result is coincident with our measurements. As we observed a positive charge at the footpads of the geckos but a negative charge with a net nominal density of $4.04 \mathrm{pC} / \mathrm{mm}^{2}$ on the acrylic materials (Fig. 2), the actual tribocharge density could be further estimated as $\sim 277.7 \mathrm{pC} / \mathrm{mm}^{2}$. Considering that the setae density at the foot of a gecko is about $14,400 \mathrm{~mm}^{-2}$ [39], a 19.3 fC tribocharge may be expected from a single seta.

\subsection{Electrostatic attraction at gecko feet}

First, we considered the electrostatic force caused by tribocharge at the foot level, similar to that in Ref. [34]. As shown in Fig. 2(c), the footpad and substrate can be simplified as two parallel flat surfaces after the edge effect and tribocharge nonuniformity are neglected [40]. Thus, we can calculate the strength of attraction $\left(F_{\mathrm{e}}\right)$ caused by the tribocharge as follows [40]:

$$
F_{\mathrm{e}}=-\frac{\sigma^{2}}{2 \varepsilon}
$$

where $\sigma=277.7 \mathrm{pC} / \mathrm{mm}^{2}$. Air was considered as the medium with relative electric inductivity of 1 .

By taking $8.854187817 \times 10^{-12} \mathrm{~F} / \mathrm{m}$ as the $\varepsilon$ in a vacuum, we finally obtained the strength of the electrostatic force as $F_{\mathrm{e}}=4.4 \mathrm{mN} / \mathrm{mm}^{2}$. Unsurprisingly, this result is smaller than that $\left(47 \mathrm{mN} / \mathrm{mm}^{2}\right)$ reported by Izadi et al. [34] by one order of magnitude. Izadi et al. [34] obtained a shear strength of $2.3 \mathrm{~N} / \mathrm{cm}^{2}$ (i.e., $23 \mathrm{mN} / \mathrm{mm}^{2}$ ) when they slid a gecko foot on a teflon AF film. As we suspected, this value is merely $1 / 6^{\text {th }}$ of that we obtained in freely running geckos. The electrostatic force they estimated was nearly twice the shear force that they measured. Thus, they concluded that the electrostatic force originating from the tribocharge, rather than other force or interactions, is the dominant source of gecko adhesion.

Presumingly, a substantial coefficient of friction $(\mathrm{CoF})$ is required to convert the normal electrostatic attraction here into shear force if there are no other interactions at the setae-substrate interface [41]. In particular, the CoF of the teflon film should be about 0.5 if the measured shear force was caused by the electrostatic attraction. However, it is generally less than 0.1, sometimes even less than 0.05 [42]. Such a conflict partly explains why geckos always fail to maneuver on surfaces made with teflon. Thus, when geckos freely climb a wall, we are prone to support that the gecko adhesion is unlikely to be completely driven by electrostatic interactions. In comparison, the $\mathrm{CoF}$ of the acrylic in our experiments was about 0.2 . Therefore, the strength of the shear force caused by the electrostatic attraction in our experiment was $0.89 \mathrm{mN} / \mathrm{mm}^{2}$, a value about $1 / 5^{\text {th }}$ of that Izadi et al. [34] determined by sliding gecko feet, and accounting for merely $0.7 \%$ of the total shear force that we measured. 


\subsection{Contribution of tribocharge to spatulae adhesion}

Gecko seta is typically hierarchical and branch into $100-1,000$ nano spatulae ( $200 \mathrm{~nm}$ in size) at the ends [39]. To further determine the contribution of the electrostatic force that emerges from the tribocharge to setae adhesion, we attempted to estimate the adhesive force of a single spatula. Assuming the densities of the setae and spatula are $\rho_{\mathrm{h}}\left(14,400 / \mathrm{mm}^{2}\right.$ here) and $\rho_{\mathrm{s}}$ (500 per seta here), respectively, the charge at a single spatula was obtained as $c=\sigma /\left(\rho_{\mathrm{h}} \rho_{\mathrm{s}}\right)=3.85 \times 10^{-17} \mathrm{C}$. Then, two models were applied to estimate the adhesion that occurs at a spatula.

1) Sphere-flat model

In this model, the spatulas were treated as particles with a diameter $(d)$ of $200 \mathrm{~nm}$, and the substrate was considered as a flat surface, which is large enough than a single spatula.

According to the Gauss theorem [40], the field strength $(E)$ of the flat substrate possessing a charge density of $\sigma$ can be written as

$$
E=-\frac{\sigma}{2 \varepsilon}
$$

when the charge of a single spatula $c$ approaching to the flat, the electrostatic adhesive force is $F_{\mathrm{c}}=-(\sigma c) / 2 \varepsilon$.

Compared with the electrostatic force, the van der Waals force naturally occurs between two surfaces in contact [19]. In this model, the van der Waals force $\left(F_{\mathrm{v}}\right)$ between a spatula with diameter $d$ and a flat substrate can be estimated as follows [19], when the effects of tribocharge are overlooked:

$$
F_{\mathrm{v}}=-\frac{H}{6 D^{2}} \frac{d}{2}
$$

where $H$ is the material-dependent Hamaker constant, and $D$ is the distance between the spatula and substrate.

Notably, Eq. (4) holds if $d>D$. As some parameters that may weaken the force are neglected, this model presents the upper limit of the electrostatic and van der Waals force.

2) Sphere-sphere model

In this model, the spatulae were also regarded as spheres, whereas the substrate was viewed as a flat surface comprising great quantities of virtual particles of the same size $(d)$ and the same amount of charge (c). Assuming (1) each spatula interacts with a virtual particle, and (2) there is no coupling, the adhesion of the tribocharge is obtained as [40]

$$
F_{c}=-K \frac{c^{2}}{(d+D)^{2}}
$$

where $K$ is the Coulomb constant that equals $8.98755179 \times 10^{9} \mathrm{Nm}^{2} / \mathrm{C}^{2}$.

In this model, the van der Waals attractive force is [19]

$$
F_{\mathrm{v}}=-\frac{H}{6 D^{2}} \frac{d}{4}
$$

Compared with the previous spherical-flat model, the coupling among virtual particles that may enhance the force was not considered. Consequently, this sphere-sphere model achieves a lower boundary of the electrostatic and van der Waals force.

Following Autumn et al. [20], the typical Hamaker constant in the current study was taken as $10^{-19} \mathrm{~J}$. By computing the above-mentioned data, we obtained the electrostatic and van der Waals force for a single spatula, as shown in Fig. 4.

The red area in Fig. 4 shows the range of $F_{\mathrm{v}}$. When the distance between the substrate and a spatula is $0.3 \mathrm{~nm}$ [16], the van der Waals force between them ranges from 9.3 to $18.5 \mathrm{nN}$. Nevertheless, the van der Waals force decreases rapidly when the distance increases to $1 \mathrm{~nm}$. If the distance is more than $2 \mathrm{~nm}$, the van der Waals force is almost zero. In comparison, the force originating from the tribocharge remains virtually unchanged when the distance increases from 0.3 to $5 \mathrm{~nm}$. The upper boundary value remains $0.6 \mathrm{nN}$, while the lower boundary value decreases from 0.33 to $0.3 \mathrm{nN}$ in the calculated distance range (the blue region in Fig. 4). As a result, the total force that includes both varies between 9.6 and $19.1 \mathrm{nN}$ when the distance between the substrate and spatula is $0.3 \mathrm{~nm}$ (Fig. 4, green area). The final force also reduces rapidly with an increase in the gap. Interestingly, the force of a spatula $(\sim 10 \mathrm{nN})$ determined by Huber et al. [43] falls into the range we found here, indicating that our model is reasonable. Moreover, based on the force estimated from a spatula, we found that the adhesion strength could be $69.1-137.7 \mathrm{mN} / \mathrm{mm}^{2}$, a 


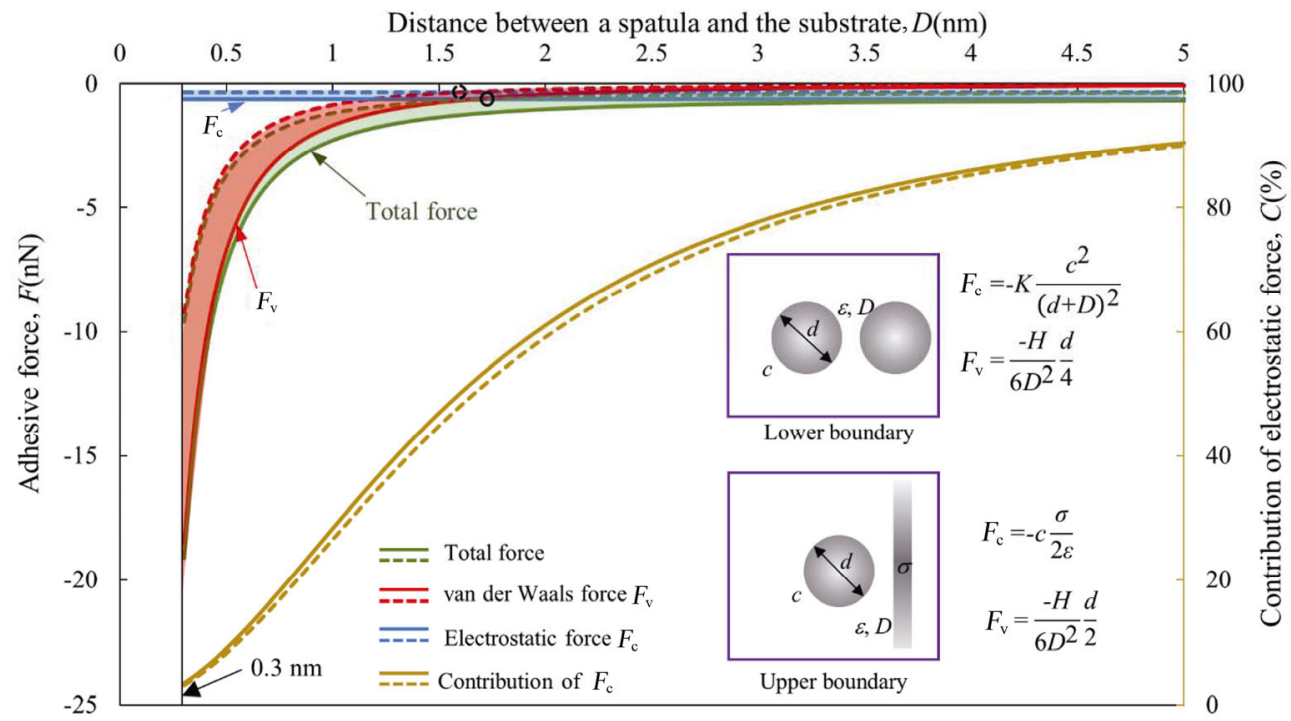

Fig. 4 Adhesion at a single spatula. The results are calculated from the models shown. The blue area shows $F_{\mathrm{c}}$, the red area shows $F_{\mathrm{v}}$, and the green area shows the total force. The contribution of electrostatic force is indicated by the yellow lines. The dashed lines indicate the lower boundaries, and the solid lines indicate the upper boundaries. The black circles illustrate the cross-points of the electrostatic and van der Waals forces. The insets show the models used for calculation. In the models, $d=$ $200 \mathrm{~nm}, c=3.85 \times 10^{-17} \mathrm{C}, \sigma=277.7 \mathrm{pC} / \mathrm{mm}^{2}, H=10^{-19} \mathrm{~J}, \varepsilon=1 \times \varepsilon_{0}=8.854187817 \times 10^{-12} \mathrm{~F} / \mathrm{m}$, and $K=8.98755179 \times 10^{9} \mathrm{~N} \cdot \mathrm{m}^{2} / \mathrm{C}^{2}$.

range within which our measured result falls.

As indicated by the black circles in Fig. 4, despite the electrostatic adhesion being smaller than the van der Waals force when the distance is $0.3 \mathrm{~nm}$, they are equal when the distance expands to about $1.6 \mathrm{~nm}(0.3 \mathrm{nN}$ for the lower boundary at $D=1.6 \mathrm{~nm}$ and $0.6 \mathrm{nN}$ for the upper limit at $D=1.65 \mathrm{~nm}$ ). The similar values of $D$ indicate that the estimations from the tow models are compatible.

After obtaining the electrostatic adhesion and van der Waals adhesion at a single spatula, we evaluated the contribution of electrostatic force by dividing it with the overall force (Fig. 4, yellow lines). No significant difference was observed between the results calculated from the upper and lower boundary models. If the distance is $0.3 \mathrm{~nm}$, the contribution of the electrostatic force to the overall force is $3.3 \%$, a value that is slightly larger than $0.7 \%$ obtained from the foot model. However, Wang and Wang [28] pointed out that the distance between two solids would be $0.2 \mathrm{~nm}$ after the occurrence of triboelectrification. At this distance, the van der Waals force will be more significant, whereas the electrostatic attraction remains almost unchanged. Therefore, the contribution of electrostatic attraction will be a bit smaller than $3.3 \%$. According to Fig. 4, although the contribution of electrostatic force may increase with the distance, the total force is rather small.

Although the value of the electrostatic force is much smaller than the maximum van der Waals attraction, the electrostatic force possesses a much longer range of function than that of the van der Waals force. Such a long action-range might be beneficial to other properties of geckos' feet, such as self-cleaning and anti-self-adhesion, encouraging us to carry out further experimental explorations on setae level or even spatulae level. Moreover, as the van der Walls force originates from polarized molecules and the electro field can affect the polarization [40], the tribocharge at geckos' feet may also contribute to adhesion through affecting the polarization, encouraging us to carry our future study from this angle.

\section{Conclusions}

In addition to the van der Waals interactions and capillary force, the electrostatic attraction was declared to be contributive to geckos adhesion, but has yet to be fully understood in running geckos. In this study, we determined that during an upward climb, the exact contact area of a gecko foot could be about $1 / 70^{\text {th }}$ of the area of the whole foot. For the 
first time, we quantitively evaluated the contribution of electrostatic forces resulting from the tribocharge occurring during free climbing to the overall frictional adhesion. The estimation at foot level showed that the tribocharge could generate an attraction of $\sim 4.4 \mathrm{mN} / \mathrm{mm}^{2}$ at a foot, causing a shear strength which accounts for about $1 \%$ of the overall shear. The results from the model analyses at the spatula level were similar to that estimated from the foot level, showing that the electrostatic attraction might contribute about $3 \%$ to the overall adhesion when the distance between a spatula and the substrate is $0.3 \mathrm{~nm}$. These findings partly explain why geckos always fail to maneuver on teflon surfaces even though they may obtain large tribocharge.

Limited by the testing techniques, we conducted analyses based on data collected from separate experiments. In the future, simultaneous measurements of the tribocharge, contact area, and reaction force may provide more accurate data. The findings also encourage us to perform experimental investigations at seta or even spatulae levels. Nevertheless, the results in this study significantly enhanced our understating of the hairy adhesion of geckos.

\section{Acknowledgements}

This study was supported by the National Natural Science Foundation of China (Grant Nos. 51435008, 51705247, and 31601870) and a Jiangsu Educational Innovation Program (Grant No. KYLX16_0327).

Open Access This article is licensed under a Creative Commons Attribution 4.0 International License, which permits use, sharing, adaptation, distribution and reproduction in any medium or format, as long as you give appropriate credit to the original author(s) and the source, provide a link to the Creative Commons licence, and indicate if changes were made.

The images or other third party material in this article are included in the article's Creative Commons licence, unless indicated otherwise in a credit line to the material. If material is not included in the article's Creative Commons licence and your intended use is not permitted by statu- tory regulation or exceeds the permitted use, you will need to obtain permission directly from the copyright holder.

To view a copy of this licence, visit http://creativecommons.org/licenses/by/4.0/.

\section{References}

[1] Chen J J, Peattie A M, Autumn K, Full R J. Differential leg function in a sprawled-posture quadrupedal trotter. $J$ Exp Biol 209(2): 249-259 (2006)

[2] Autumn K, Hansen W. Ultrahydrophobicity indicates a non-adhesive default state in gecko setae. J Comp Physiol A 192(11): 1205-1212 (2006)

[3] Wang Z Y, Wang J T, Ji A H, Zhang Y Y, Dai Z D. Behavior and dynamics of gecko's locomotion: The effects of moving directions on a vertical surface. Chinese Sci Bull 56(6): 573-583 (2011)

[4] Wang Z Y, Dai Z D, Li W, Ji A H, Wang W B. How do the substrate reaction forces acting on a gecko's limbs respond to inclines? Sci Nat 102(1): 7 (2015)

[5] Song Y, Dai Z D, Wang Z Y, Full R J. Role of multiple, adjustable toes in distributed control shown by sideways wall-running in geckos. Proc $R$ Soc B Biol Sci 287: 20200123 (2020).

[6] Autumn K, Puthoff J. Properties, principles, and parameters of the gecko adhesive system. In Biological Adhesives. Smith A M, Ed. Cham: Springer, 2016: 245280.

[7] Russell A P, Bauer A M, Laroiya R. Morphological correlates of the secondarily symmetrical pes of gekkotan lizards. J Zool 241(4): 767-790 (1997)

[8] Russell A P, Stark A Y, Higham T E. The integrative biology of gecko adhesion: historical review, current understanding, and grand challenges. Integr Comp Biol 59(1): 101-116 (2019)

[9] Home E. IX. Some account of the feet of those animals whose progressive motion can be carried on in opposition to gravity. Philos Trans R Soc London 106: 149-155 (1816)

[10] Schmidt H. Zur anatomie und physiologie der geckopfote. Jena Z Naturwiss 39: 551-580 (1904)

[11] Hora S L. The adhesive apparatus on the toes of certain geckos and tree frogs. J Proc Asiat Soc Bengal 19: 138-145 (1923)

[12] Mahendra B C. Contributions to the bionomics, anatomy, reproduction and development of the indian housegecko, Hemidactylus flaviviridis Rüppel, Part II: The problem of locomotion. Proc Indian Acad Sci Sect B 13(5): 288-306 (1941)

[13] Cartier O. Studien über den feineren Bau der Haut bei 
den Reptilien. I Die Epidermis der Geckotiden. Arb Zool Inst Würzburg 1: 83-96 (1872)

[14] Dellit W D. Zur anatomie und physiologie der Geckozehe. Jena Z Naturwiss 68: 613-656 (1934)

[15] Maderson P F A. Keratinized epidermal derivatives as an aid to climbing in Gekkonid Lizards. Nature 203(4946): 780-781 (1964)

[16] Autumn K, Sitti M, Liang Y A, Peattie A M, Hansen W R, Sponberg S, Kenny T W, Fearing R, Israelachvili J N, Full R J. Evidence for van der Waals adhesion in gecko setae. Proc Natl Acad Sci USA 99(19): 12252-12256 (2002)

[17] Haase A. Untersuchungen über den bau und die entwicklung der haftlappen bei den geckotiden. Arch Naturgesch 66: 321-345 (1900).

[18] Hiller U. Untersuchungen zum feinbau und zur funktion der haftborsten von reptilien. Z Morphol Tiere 62(4): 307-362 (1968)

[19] Israelachvili J N. Intermolecular and Surface Forces. 3rd edn. Singapore: Elsevier, 2012.

[20] Autumn K, Liang Y A, Hsieh S T, Zesch W, Chan W P, Kenny T W, Fearing R, Full R J. Adhesive force of a single gecko foot-hair. Nature 405(6787): 681-685 (2000)

[21] Huber G, Mantz H, Spolenak R, Mecke K, Jacobs K, Gorb S N, Arzt E. Evidence for capillarity contributions to gecko adhesion from single spatula nanomechanical measurements. Proc Natl Acad Sci USA 102(45): 16293-16296 (2005).

[22] Puthoff J B, Prowse M S, Wilkinson M, Autumn K. Changes in materials properties explain the effects of humidity on gecko adhesion. $J$ Exp Biol 213(21): 3699-3704 (2010)

[23] Prowse M S, Wilkinson M, Puthoff J B, Mayer G, Autumn K. Effects of humidity on the mechanical properties of gecko setae. Acta Biomater 7(2): 733-738 (2011)

[24] Chen B, Gao H. An alternative explanation of the effect of humidity in gecko adhesion: Stiffness reduction enhances adhesion on a rough surface. Int J Appl Mech 2(01): 1-9 (2010).

[25] Alibardi L. Ultrastructural autoradiographic and immunocytochemical analysis of setae formation and keratinization in the digital pads of the gecko Hemidactylus turcicus (Gekkonidae, Reptilia). Tissue Cell 35(4): 288-296 (2003)

[26] Zou H Y, Zhang Y, Guo L T, Wang P H, He X, Dai G Z, Zheng $\mathrm{H} \mathrm{W}$, Chen $\mathrm{C} \mathrm{Y}$, Wang A C, Xu C, et al. Quantifying the triboelectric series. Nat Commun 10(1): 1427 (2019)
[27] Lee B W, Orr D E. The triboelectric series. https://www. alphalabinc.com/triboelectric-series, 2009.

[28] Wang Z L, Wang A C. On the origin of contactelectrification. Mater Today 30: 34-51 (2019)

[29] Lacks D J, Sankaran R M. Contact electrification of insulating materials. $J$ Phys D Appl Phys 44(45): 453001 (2011)

[30] Baytekin H T, Patashinski A Z, Branicki M, Baytekin B, Soh S, Grzybowski B A. The mosaic of surface charge in contact electrification. Science 333(6040): 308-312 (2011)

[31] Lowell J, Truscott W S. Triboelectrification of identical insulators. II. Theory and further experiments. $J$ Phys $D$ Appl Phys 19(7): 1281 (2000)

[32] Prevenslik T. Electrostatic gecko mechanism. Tribol Ind 31(1-2): 61-66 (2009)

[33] Izadi H, Sarikhani K, Penlidis A. Instabilities of teflon AF thin films in alumina nanochannels and adhesion of bi-level Teflon AF nanopillars. Nanotechnology 24(50): 505306 (2013)

[34] Izadi H, Stewart K M E, Penlidis A. Role of contact electrification and electrostatic interactions in gecko adhesion. J Roy Soc Interface 11(98): 20140371 (2014)

[35] The editors of Encyclopaedia Britannica. Surface tension. https://www.britannica.com/science/surface-tension, 2015.

[36] Song Y, Wang Z Y, Zhou J, Li Y, Dai Z D. Synchronous measurement of tribocharge and force at the footpads of freely moving animals. Friction 6(1): 75-83 (2018)

[37] Eason E V, Hawkes E W, Windheim M, Christensen D L, Libby T, Cutkosky M R. Stress distribution and contact area measurements of a gecko toe using a high-resolution tactile sensor. Bioinspir Biomim 10(1): 016013 (2015)

[38] Huber G, Gorb S N, Hosoda N, Spolenak R, Arzt E. Influence of surface roughness on gecko adhesion. Acta Biomater 3(4): 607-610 (2007)

[39] Ruibal R, Ernst V. The structure of the digital setae of lizards. J Morphol 117(3): 271-293 (1965)

[40] Cheng D K. Field and Wave Electromagnetics. 2nd edn. Reading (USA): Prentice Hall, 1989.

[41] Cutnell J D, Johnson K W, Young D, Stadler S, Young Sr D. Physics. 10 ${ }^{\text {th }}$ edn. New York (USA): Wiley, 2014.

[42] Sastri V R. Plastics in Medical Devices: Properties, Requirements, and Applications. New York (USA): Elsevier, 2010.

[43] Huber G, Gorb S N, Spolenak R, Arzt E. Resolving the nanoscale adhesion of individual gecko spatulae by atomic force microscopy. Biol Lett 1(1): 2-4 (2005) 


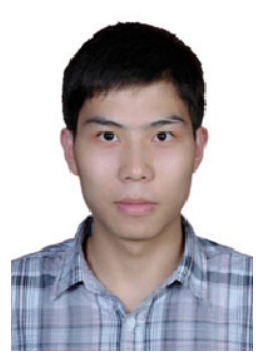

Yi SONG. He obtained his Ph.D. degree in 2020 from the College of Mechanical and Electrical Engineering, Nanjing University of Aeronautics and Astronautics

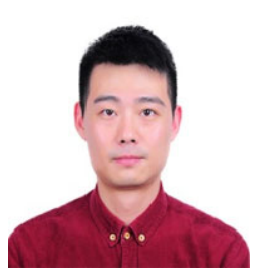

Zhouyi WANG. He obtained his Ph.D. degree in 2015 from the College of Mechanical and Electrical Engineering at NUAA. He was awarded the Bronze Prize of the

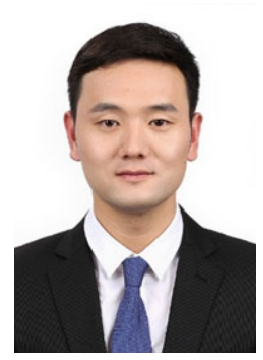

Yang LI. He received his Ph.D. degree in 2016 from the College of Materials Science and Technology at NUAA, China. He is carrying out his postdoctoral research in

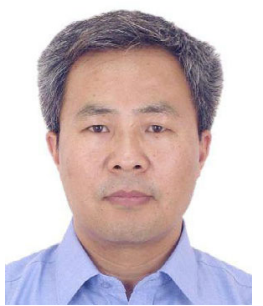

Zhendong DAI. He obtained his Ph.D. degree in 1999 from the College of Mechanical and Electrical Engineering at NUAA, and now is a professor in mechanical engineering at NUAA, China. He is one of the Chinese delegates of the International Society of Bionic Engineering, an
(NUAA), China. He is currently continuing his postdoctoral research at NUAA. His research interest involves bio-tribology, biomechanics, and bio-inspired designs.

Shangyin Excellent Mechanical Doctoral Dissertation Award in 2016. He is an associate professor at NUAA. His interested research areas include tribology, bionics, and animal kinematics and dynamics.

the College of Mechanical and Electrical Engineering at NUAA. His research interests include bioinspired materials, surface friction, micro-nano structural design, and carbon nanotube materials.

executive member of the council of Chinese Mechanical Engineering in Tribology. He is on the academic committee board of State Key Laboratory of Solid Lubrication, and on the editorial boards of many magazines such as the Journal of Bionic Engineering, International Journal of Vehicle Autonomous System, Friction, etc. His research interests are tribology and bio-inspired materials and robots. 